\title{
Betatron frequency and the Poincaré rotation number
}

\author{
Sergei Nagaitsev(1,2 and Timofey Zolkin $\oplus^{1}$ \\ ${ }^{1}$ Fermilab, Batavia, Illinois 60510, USA \\ ${ }^{2}$ The Enrico Fermi Institute, The University of Chicago, Chicago, Illinois 60637, USA
}

(Received 2 November 2019; accepted 13 April 2020; published 8 May 2020)

\begin{abstract}
Symplectic maps are routinely used to describe single-particle dynamics in circular accelerators. In the case of a linear accelerator map, the rotation number (the betatron frequency) can be easily calculated from the map itself. In the case of a symplectic nonlinear map, the rotation number is normally obtained numerically, by iterating the map for given initial conditions, or through a perturbation approach. Integrable maps, a subclass of symplectic maps, allow for an analytic evaluation of their rotation numbers. In this paper we propose an analytic expression to determine the rotation number for integrable symplectic maps of the plane, if an integral is explicitly known, and present several examples, relevant to accelerators. If the integral of motion is not explicitly known, one can obtain the rotation number numerically as outlined in Appendix B. These new results can be used to analyze the topology of the accelerator Hamiltonians as well as to serve as the starting point for a perturbation theory for maps.
\end{abstract}

DOI: 10.1103/PhysRevAccelBeams.23.054001

\section{INTRODUCTION}

The first mention of the betatron frequency was in the 1941 pioneering work by Kerst and Serber [1], where they defined it as the fractional number of particle oscillations around the orbit per one revolution period in a betatron (a type of an induction accelerator). Later, the theory of the alternating-gradient (AG) synchrotron [2] demonstrated the existence of an integral of motion (the so-called CourantSnyder invariant) for particles in an AG synchrotron and established a powerful connection between the modern AG focusing systems and linear symplectic maps, thus connecting the betatron frequency and the Poincaré rotation number [3].

In modern accelerators (for example, in the LHC) particles are stored for up to $10^{8}-10^{9}$ revolutions and understanding their dynamics is crucially important for maintaining long-term particle stability $[4,5]$. One important parameter of particle dynamics in an accelerator is the betatron frequency and its dependence on a particle's amplitude. It turns out that the accelerator focusing systems conserve the Courant-Snyder invariant only approximately and there is a need to analyze the conditions for stable particle dynamics. Over the recent years, several methods were developed to analyze the particle motion in accelerator systems, using either numeric tools, like the

Published by the American Physical Society under the terms of the Creative Commons Attribution 4.0 International license. Further distribution of this work must maintain attribution to the author(s) and the published article's title, journal citation, and DOI.
Frequency Map Analysis [6], or the Normal Form Analysis [7,8], an extension of the Jordan normal form analysis for matrices.

At the same time, there has been continuous interest, starting with McMillan [9], in making the accelerator maps nonlinear, yet integrable [10-13]. However, there does not exist an analytic method to calculate the betatron frequency (the Poincare rotation number) for nonlinear symplectic integrable maps. This present paper is set to remedy this deficiency.

\section{BETATRON FREQUENCY}

For a 1 degree-of-freedom time-independent system, the Hamiltonian function, $\mathrm{H}[p, q ; t]=\mathrm{E}$, is the integral of the motion. If the motion is bounded, it is also periodic, and the period of oscillations can be determined by integrating

$$
T(\mathrm{E})=\oint\left(\frac{\partial \mathrm{H}}{\partial p}\right)^{-1} \mathrm{~d} q,
$$

where $p=p(\mathrm{E}, q)$ [14]. The oscillation period and its dependence on initial conditions is one of the key properties of the periodic motion. Typically, a stroboscopic map for an autonomous (time-independent) system can be constructed to analyze this periodic motion, by evaluating the phase-space coordinates $(q, p)$ at periodic time intervals, $t_{k}=k \tau$ with $k=0,1,2, \ldots$ (see, for example, [15]). Knowing the period $T(E)$, one can calculate the Poincaré rotation number for such a map as $\nu(E)=\tau / T(E)$.

Modern high-energy accelerators can be described as nonautonomous systems in 2 or 3 degrees of freedom, with 
the Hamiltonian depending periodically on an independent variable, $s$, which is the longitudinal coordinate along the reference trajectory [16]. Because the system is periodic, it is natural to use one accelerator revolution period as the stroboscopic map's time interval, $\tau$ [17]. Most of the present-day accelerator designs start with a linear focusing system, where one needs to determine $T(E)$ only once, in order to obtain the rotation number for all particles, regardless of their amplitudes. Nonlinear focusing elements (e.g., sextupoles and octupoles) are then added to mitigate various effects, making the resulting system nonlinear. When the accelerator Hamiltonian is nonlinear yet periodic, calculating $T(E)$ for each particle by direct tracking may be time consuming. Below we propose an analytic expression to determine the rotation number for accelerator maps of the plane, if an integral of motion is explicitly known. This integral of motion is typically not the Hamiltonian function, which is nonautonomous yet periodic.

Let us now consider a symplectic map of the plane (corresponding to a one-turn map of an accelerator), $\mathrm{M}: \mathbb{R}^{2} \rightarrow \mathbb{R}^{2}$,

$$
\left(q^{\prime}, p^{\prime}\right)=\mathrm{M}(q, p),
$$

where the prime symbols $\left({ }^{\prime}\right)$ indicate the transformed phase space coordinates. Suppose that the sequence, generated by a repeated application of the map,

$$
\left(q_{0}, p_{0}\right) \rightarrow\left(q_{1}, p_{1}\right) \rightarrow\left(q_{2}, p_{2}\right) \rightarrow\left(q_{3}, p_{3}\right) \rightarrow \cdots,
$$

belongs to a closed invariant curve. We do not describe how this map is obtained (see, for example, $[17,18]$ ) but let us suppose that we know the mapping equations. Let $R_{n}$ be the rotation angle in the phase space $(q, p)$ around a stable fixed point between two consecutive iterations $\left(q_{n}, p_{n}\right)$ and $\left(q_{n+1}, p_{n+1}\right)$. Then, the limit, when it exists,

$$
\nu=\lim _{N \rightarrow \infty} \frac{1}{2 \pi N} \sum_{n=0}^{N} R_{n}
$$

is called the rotation number (the betatron frequency of the one-turn map) for that particular orbit of the map M [19,20]. Unlike Eq. (1), which allows to express the oscillation period analytically, Eq. (2) can be only evaluated numerically for each orbit. Let us now suppose that there exists a nonconstant real-valued continuous function $\mathcal{K}(q, p)$, which is invariant under $\mathrm{M}$. The function $\mathcal{K}(q, p)$ is called integral and the map is called integrable. In this paper, we are describing the case, for which the level sets $\mathcal{K}=$ const are compact closed curves (or sets of points) and for which the identity

$$
\mathcal{K}\left(q^{\prime}, p^{\prime}\right)=\mathcal{K}(q, p)
$$

holds for all $(q, p)$. There are many examples of integrable maps, including the famous McMillan map [9], described below. The dynamics is in many ways similar to that of a continuous system, however, the integral $\mathcal{K}(q, p)$ is not the Hamiltonian function. Thus, even though the accelerator revolution period, $\tau$, is known, the integral $\mathcal{K}(q, p)$ cannot be used in Eq. (1). Below, we will present an expression (the Danilov equation) to obtain the rotation number from $\mathcal{K}(q, p)$ for an integrable map, $\mathrm{M}$.

The Arnold-Liouville theorem for integrable maps [21-23] states that (1) the action-angle variables exist and (2) in these variables, consecutive iterations of integrable map M lie on nested circles of radius $J$ and that the map can be written in the form of a twist map,

$$
\left[\begin{array}{l}
J_{n+1} \\
\theta_{n+1}
\end{array}\right]=\left[\begin{array}{rl}
J_{n} & \\
\theta_{n}+2 \pi \nu(J) & \bmod 2 \pi
\end{array}\right],
$$

where $|\nu(J)| \leq 0.5$ is the rotation number, $\theta$ is the angle variable and $J$ is the action variable, defined as

$$
J(\mathcal{K})=\frac{1}{2 \pi} \oint p(\mathcal{K}, q) \mathrm{d} q .
$$

One can notice that the new mapping (4) is symplectic for any value of an unknown rotation number, $\nu(J)$. Unlike the action variable, $J$, the knowledge of the integral $\mathcal{K}$ alone is not sufficient to define the angle variable and to determine the rotation number. Thus, in this paper, we would like to consider the following question: how does one determine the rotation number, $\nu(\mathcal{K})$, from the known integral, $\mathcal{K}(q, p)$, and the known integrable map, M? In addition, in the "Examples" section we propose how to use this equation when only an approximate invariant is known.

\section{DANILOV EQUATION}

Danilov equation [24]: Suppose a symplectic map of the plane,

$$
\left(q^{\prime}, p^{\prime}\right)=\mathrm{M}(q, p),
$$

is integrable with the invariant (integral) $\mathcal{K}(q, p)$, then its Poincaré rotation number is

$$
\nu(\mathcal{K})=\int_{q}^{q^{\prime}}\left(\frac{\partial \mathcal{K}}{\partial p}\right)^{-1} \mathrm{~d} q / \oint\left(\frac{\partial \mathcal{K}}{\partial p}\right)^{-1} \mathrm{~d} q
$$

where the integrals are evaluated along the invariant curve, $\mathcal{K}(q, p)$.

Proof.-Consider the following system of differential equations:

$$
\frac{\mathrm{d} Q}{\mathrm{~d} t}=\frac{\partial \mathcal{K}(Q, P)}{\partial P}, \quad \frac{\mathrm{d} P}{\mathrm{~d} t}=-\frac{\partial \mathcal{K}(Q, P)}{\partial Q} .
$$

We notice that $\mathcal{K}(Q, P)$ does not change along a solution of the system, because it is an integral of the motion, meaning 

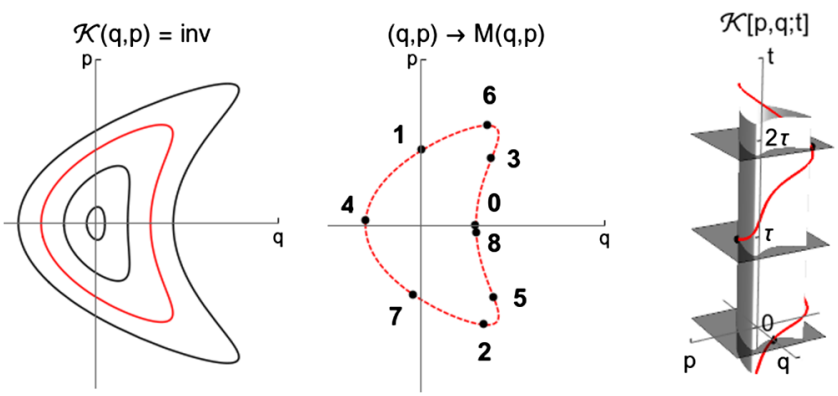

FIG. 1. Constant level sets of the integral $\mathcal{K}(q, p)=\mathrm{const}$ (left). A particular curve representing a level set of $\mathcal{K}$ and several iterates of the map $\mathrm{M}$ (center). A three-dimensional phase space, $(q, p)+$ time, of the system (7) (right). Dark gray planes $t=$ $0, \tau, 2 \tau, \ldots$ represent the stroboscopic Poincaré section of the continuous flow of the system (red curve) which is identical to map $\mathrm{M}$.

$$
\frac{\mathrm{d} \mathcal{K}}{\mathrm{d} t}=\frac{\partial \mathcal{K}}{\partial Q} \frac{\mathrm{d} Q}{\mathrm{~d} t}+\frac{\partial \mathcal{K}}{\partial P} \frac{\mathrm{d} P}{\mathrm{~d} t}=0
$$

for any solution $Q(t)$ and $P(t)$. Let $q(t)$ and $p(t)$ be the solutions of the system (7) with the following initial conditions $q(0)=q_{0}$ and $p(0)=p_{0}$. Define a new map, $\tilde{\mathrm{M}}(q, p)$ (see Fig. 1):

$$
\left(q^{\prime}, p^{\prime}\right)=\tilde{\mathrm{M}}(q, p)=[q(\tau), p(\tau)],
$$

where $\tau$ is a discrete time step. For a given $\mathcal{K}$, which is an integral of both $\mathrm{M}$ and $\tilde{\mathrm{M}}$, one can always select $\tau(\mathcal{K})$ such that the maps $\mathrm{M}(q, p)$ and $\tilde{\mathrm{M}}(q, p)$ are identical. This follows from the Arnold-Liouville theorem. Since $\mathcal{K}(q, p)$ is compact and closed, the functions $q(t)$ and $p(t)$ are periodic with a period $T(\mathcal{K})$. By its definition,

$$
\tau=\nu(\mathcal{K}) T(\mathcal{K}) .
$$

Let us now calculate $\nu(\mathcal{K})$ :

$\nu(\mathcal{K}) \equiv \frac{\tau}{T}=\frac{\int_{q}^{q^{\prime}} \mathrm{d} t}{\oint \mathrm{d} t}=\frac{\int_{q}^{q^{\prime}}\left(\frac{\mathrm{d} q}{\mathrm{~d} t}\right)^{-1} \mathrm{~d} q}{\oint\left(\frac{\mathrm{d} q}{\mathrm{~d} t}\right)^{-1} \mathrm{~d} q}=\frac{\int_{q}^{q^{\prime}}\left(\frac{\partial \mathcal{K}}{\partial p}\right)^{-1} \mathrm{~d} q}{\oint\left(\frac{\partial \mathcal{K}}{\partial p}\right)^{-1} \mathrm{~d} q}$.

Q.E.D.

Corollary 0.1.-

$$
\nu(\mathcal{K})=\frac{\mathrm{d} J^{\prime}}{\mathrm{d} J}
$$

where

$$
J^{\prime}(\mathcal{K})=\frac{1}{2 \pi} \int_{q}^{q^{\prime}} p(\mathcal{K}, q) \mathrm{d} q
$$

is the partial action calculated as a sector integral (see Fig. 2) around the stable fixed point.
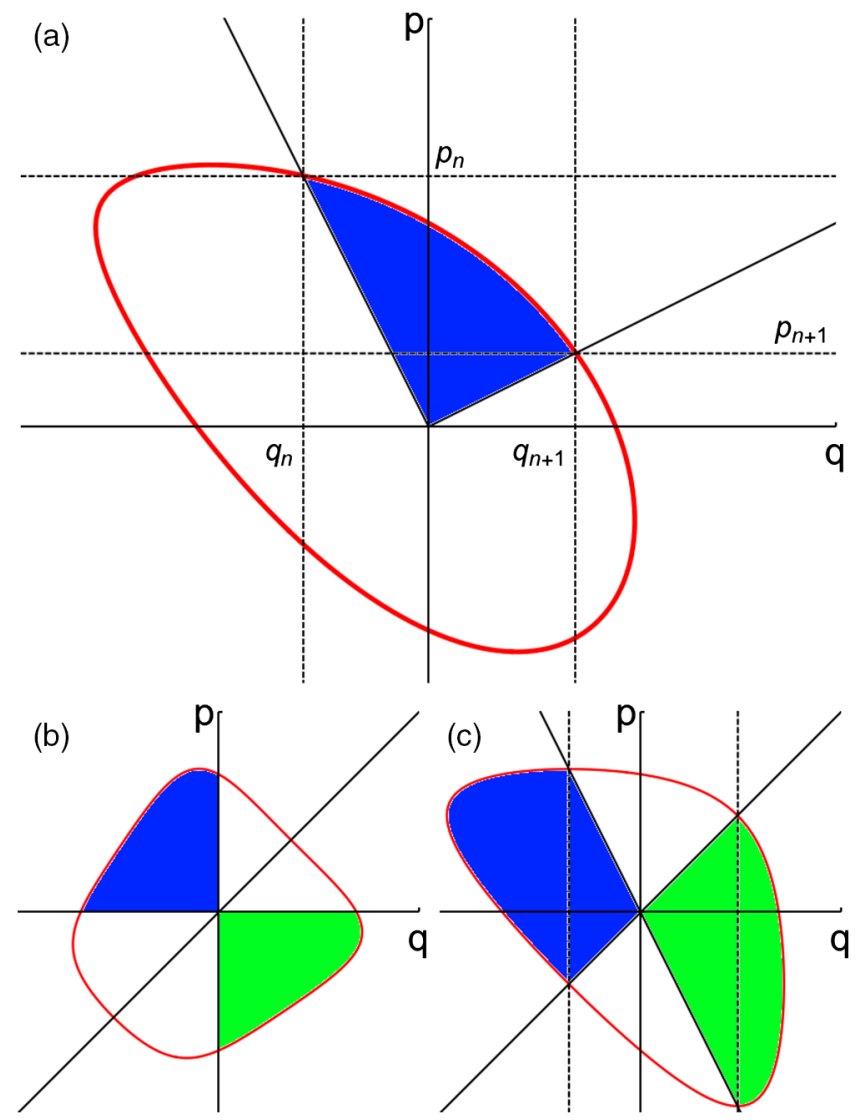

FIG. 2. The partial action is defined as a sector area (blue) for one map iteration, divided by $2 \pi$ (a). Convenient choices of the partial action for mappings in McMillan form: an area under the curve in II (blue) or IV (green) quadrants (b), and areas for initial conditions in a form of $\left(q_{0}, q_{0}\right)$ (c).

Proof.-First, we will consider the denominator in Eq. (11):

$$
\frac{1}{2 \pi} \oint\left(\frac{\partial \mathcal{K}}{\partial p}\right)^{-1} \mathrm{~d} q=\frac{1}{2 \pi} \frac{\mathrm{d}}{\mathrm{d} \mathcal{K}} \oint p \mathrm{~d} q=\frac{\mathrm{d} J}{\mathrm{~d} \mathcal{K}}
$$

Second, we will evaluate the numerator. Using the equations of motion in Eq. (7), we notice that

$$
\int_{q}^{q^{\prime}}\left(\frac{\partial \mathcal{K}}{\partial p}\right)^{-1} \mathrm{~d} q=-\int_{p}^{p^{\prime}}\left(\frac{\partial \mathcal{K}}{\partial q}\right)^{-1} \mathrm{~d} p
$$

Now, we will utilize the Leibniz integral rule together with Eq. (15) to obtain

$$
\begin{aligned}
& \frac{1}{2 \pi} \int_{q}^{q^{\prime}}\left(\frac{\partial \mathcal{K}}{\partial p}\right)^{-1} \mathrm{~d} q \\
& \quad=\frac{1}{2 \pi} \times \frac{\mathrm{d}}{\mathrm{d} \mathcal{K}}\left(\frac{q p-q^{\prime} p^{\prime}}{2}+\int_{q}^{q^{\prime}} p \mathrm{~d} q\right)=\frac{\mathrm{d} J^{\prime}}{\mathrm{d} \mathcal{K}}
\end{aligned}
$$


Finally, by combining Eqs. (14) and (16) we obtain Eq. (12).

Corollary 0.2.-For a linear map $(\nu=$ const $)$,

$$
\nu=J^{\prime} / J
$$

Proof.-Since $\nu=$ const, the Hamiltonian function is $\mathrm{H}(J)=\nu J$. Using Eq. (12), we obtain Eq. (17).

Corollary 0.3.-The Hamiltonian function corresponding to the map $M$ is

$$
\mathrm{H}(\mathcal{K})=J^{\prime}(\mathcal{K})
$$

Proof.-Since $\nu=\mathrm{dH} / \mathrm{d} J$, one can use Eq. (12) to obtain $\mathrm{H}=J^{\prime}+$ const.

Corollary 0.4.-

$$
\nu(\mathcal{K})=\int_{p}^{p^{\prime}}\left(\frac{\partial \mathcal{K}}{\partial q}\right)^{-1} \mathrm{~d} p / \oint\left(\frac{\partial \mathcal{K}}{\partial q}\right)^{-1} \mathrm{~d} p
$$

where the integrals are evaluated along the invariant curve, $\mathcal{K}(q, p)$.

Proof.-Because of the $p \leftrightarrow-q$ symmetry in Eq. (7), the proof is similar to Eq. (11).

In order to generalize the Danilov equation to higherdimensional integrable maps, one has to know the variables, where such a map is separated into maps for each degree of freedom. Below we will consider an example of a 4D map, which is separable in polar coordinates with two integrals of motion.

\section{EXAMPLES}

In order to employ this equation in practice, one would need to recall that with $p=p(\mathcal{K}, q)$, the integrand in Eq. (6) is

$$
\left(\frac{\partial \mathcal{K}}{\partial p}\right)^{-1}=\frac{\partial p(\mathcal{K}, q)}{\partial \mathcal{K}}
$$

Also, the lower limit of the integral can be chosen to be any convenient value of $q$, for example 0 , as long it belongs to a given level set, $\mathcal{K}(q, p)$. Finally, the upper limit of the integral, $q^{\prime}$, is obtained from the selected $q$ and $p=$ $p(\mathcal{K}, q)$ by iterating the map, $\mathrm{M}(q, p)$. It is clear that not all functions $\mathcal{K}(q, p)$ can be inverted analytically to obtain $p=p(\mathcal{K}, q)$. The drawback of this method can be overcome by numeric evaluations (see the Appendix B).

For maps in a special (McMillan) form [9],

$$
\left[\begin{array}{c}
q^{\prime} \\
p^{\prime}
\end{array}\right]=\left[\begin{array}{c}
p \\
-q+f(p)
\end{array}\right],
$$

the convenient choices for integration limits in Eq. (6) are $(q, p)=\left(q_{0}, 0\right)$ and $\left(q^{\prime}, p^{\prime}\right)=\left(0,-q_{0}+f(0)\right)$, Fig. 2(b), and $(q, p)=(a, a)$ and $\left(q^{\prime}, p^{\prime}\right)=(a,-a+f(a))$, Fig. 2(c).
Finally, for twist maps, Eq. (4), the Danilov equation Eq. (6) gives $\nu$, as expected.

Let us now consider several nontrivial examples. Linear maps are presented in Appendix A.

\section{A. McMillan map}

As our first example, we will consider the so-called McMillan map [9],

$$
\left[\begin{array}{c}
q^{\prime} \\
p^{\prime}
\end{array}\right]=\left[\begin{array}{c}
p \\
-q+a p /\left(b p^{2}+1\right)
\end{array}\right] .
$$

This map has been considered in detail in Refs. [25,26]. To illustrate the Danilov equation, we will limit ourselves to a case with $b>0$ and $|a|<2$, which corresponds to stable motion at small amplitudes. Mapping (22) has the following integral:

$$
\mathcal{K}(q, p)=b q^{2} p^{2}+q^{2}+p^{2}-a q p,
$$

which is non-negative for the chosen parameters.

We first notice that for small amplitudes, $b p^{2} \ll 1$, this map can be approximated as

$$
\left[\begin{array}{c}
q^{\prime} \\
p^{\prime}
\end{array}\right] \approx\left[\begin{array}{c}
p \\
-q+a p-a b p^{3}+a b^{2} p^{5}-\cdots
\end{array}\right],
$$

and its zero-amplitude rotation number is [2]

$$
\nu(0)=\frac{1}{2 \pi} \arccos \frac{a}{2} .
$$

At large amplitudes $\left(b p^{2} \gg 1\right)$, the rotation number becomes 0.25 . We will now evaluate the rotation number analytically, using Eq. (6): Let us define a parameter,

$$
w(\mathcal{K})=\frac{1}{\sqrt{2}} \sqrt{1+\frac{d(\mathcal{K})}{\sqrt{d(\mathcal{K})^{2}+4 \mathcal{K} b}}},
$$

which spans from 0 to 1 and where $d(\mathcal{K})=a^{2} / 4+\mathcal{K} b-1$. Then, the rotation number can be expressed through Jacobi elliptic functions as follows:

$$
\nu(\mathcal{K})=\frac{1}{4 \mathrm{~K}(w)} \operatorname{arcds}\left\{\left[d(\mathcal{K})^{2}+4 \mathcal{K} b\right]^{-1 / 4}, w\right\},
$$

where $\mathrm{K}(w)$ is the complete elliptic integral of the first kind and the inverse Jacobi function, $\operatorname{arcds}(x, w)$, is defined as follows:

$$
\operatorname{arcds}(x, w)=\int_{x}^{\infty} \frac{\mathrm{d} t}{\sqrt{\left(t^{2}+w^{2}\right)\left(t^{2}+w^{2}-1\right)}} .
$$



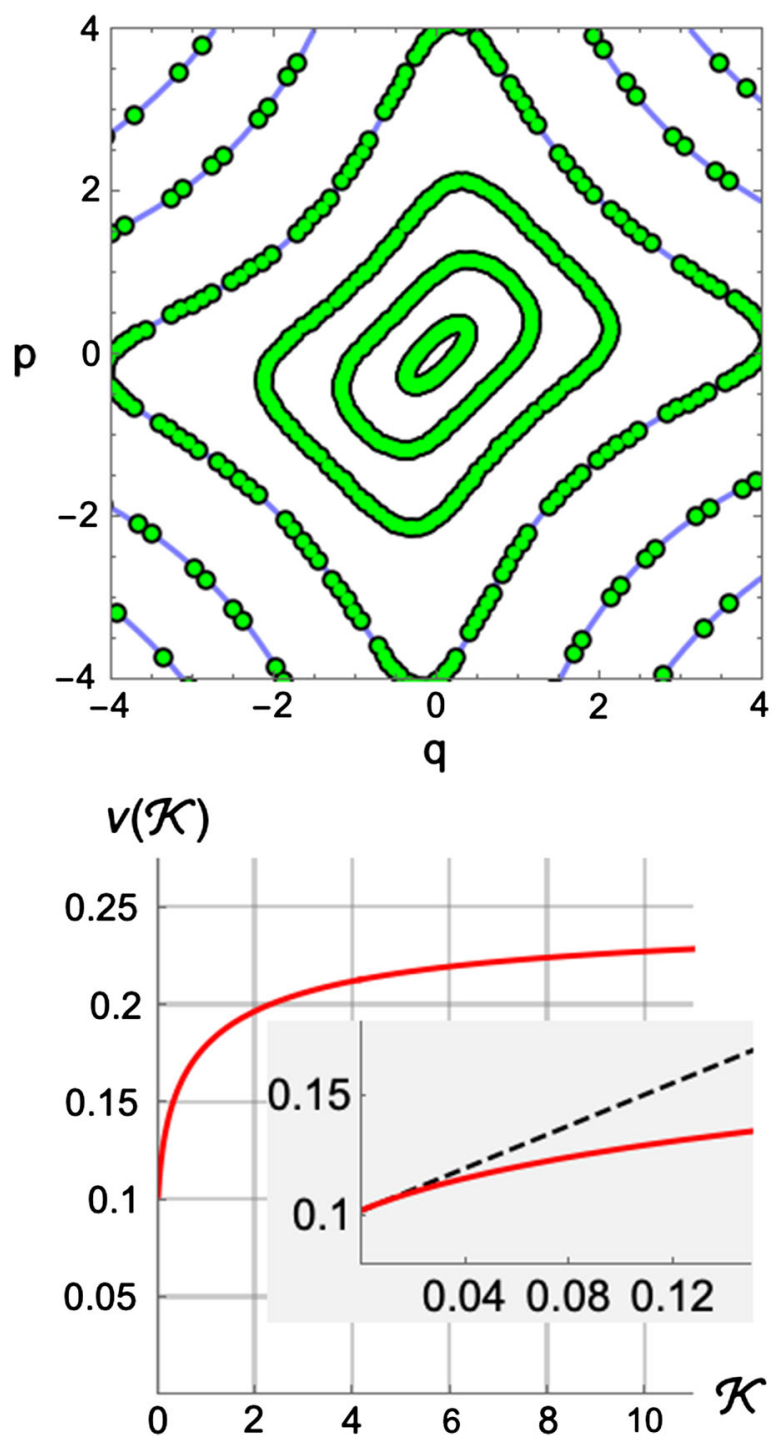

FIG. 3. The top plot contains iterations (green dots) of the McMillan map $(a=1.6, b=1)$. Constant level sets of the invariant are shown with blue lines. The bottom plot is the rotation number, Eq. (27), as a function of its integral, $\mathcal{K}$. The inset shows the linear approximation, Eq. (29).

The rotation number, Eq. (27), has the following series expansion:

$$
\nu(\mathcal{K}) \approx \nu(0)+\frac{3}{2 \pi} \frac{b a}{\sqrt{\left(4-a^{2}\right)^{3}}} \mathcal{K} .
$$

Figure 3 shows an example of the rotation number, for the case of $a=1.6$ and $b=1(\nu(0) \approx 0.102)$, as a function of integral, $\mathcal{K}$.

The McMillan invariant (23) also allows for an analytic evaluation of the action integral (5). We will omit the lengthy expressions, but will only present a smallamplitude series expansion:

$$
J(\mathcal{K}) \approx \frac{\mathcal{K}}{\sqrt{4-a^{2}}}-\frac{b\left(2+a^{2}\right) \mathcal{K}^{2}}{\sqrt{\left(4-a^{2}\right)^{5}}}
$$

Finally, we can also present a small-amplitude series expansion of the rotation number (27):

$$
\nu(J) \approx \nu(0)+\frac{3}{2 \pi} \frac{b a}{4-a^{2}} J .
$$

\section{B. Cubic map}

As our second example, we will consider a nonintegrable Hénon cubic map [27,28]:

$$
\left[\begin{array}{c}
q^{\prime} \\
p^{\prime}
\end{array}\right]=\left[\begin{array}{c}
p \\
-q+a p+\epsilon p^{3}
\end{array}\right] .
$$

This map is well known in accelerator physics as a symplectic octupole map. At small amplitudes this map is linear and the rotation number is

$$
\nu \approx \frac{1}{2 \pi} \arccos \left(\frac{a}{2}\right)
$$

At large amplitudes this map becomes chaotic and unstable. Let us propose an approximate integral (the exact integral does not exist since it is a nonintegrable map):

$$
\begin{aligned}
\mathcal{K}_{\mathrm{c}}(q, p)= & p^{2}+q^{2}-a p q-\frac{\epsilon}{a} p^{2} q^{2} \\
& +\frac{7 \epsilon}{5 a\left(4-a^{2}\right)}\left(p^{2}+q^{2}-a p q\right)^{2}+O\left(\epsilon^{2}\right) .
\end{aligned}
$$

The derivation of this approximate integral goes beyond the scope of this article and will be described in subsequent publications. For this illustration, the reader can verify by inspection that this integral is approximately conserved, near the origin. We will now use the Danilov equation to evaluate the rotation number of this map for various initial conditions with $q_{0}=p_{0}$. Figure 4 shows the exact (numeric), Eq. (2), and the approximate rotation number, calculated from (34) and (32) using the Danilov equation, Eq. (6). The inset at the bottom left is a magnified portion of the same plot. This is to illustrate an excellent correspondence between the orange curve, obtained by using the Danilov equation, Eq. (12) numerically for closed nonresonant phase-space trajectories, and the black curve, obtained directly from Eq. (2). Since the black curve is obtained by a direct averaging method, one may observe steplike features and mode locking, when crossing a chaotic region or a chain of islands. This is not a numerical artifact, but it is not present in the orange curve.

A small-amplitude series expansion of the rotation number is 

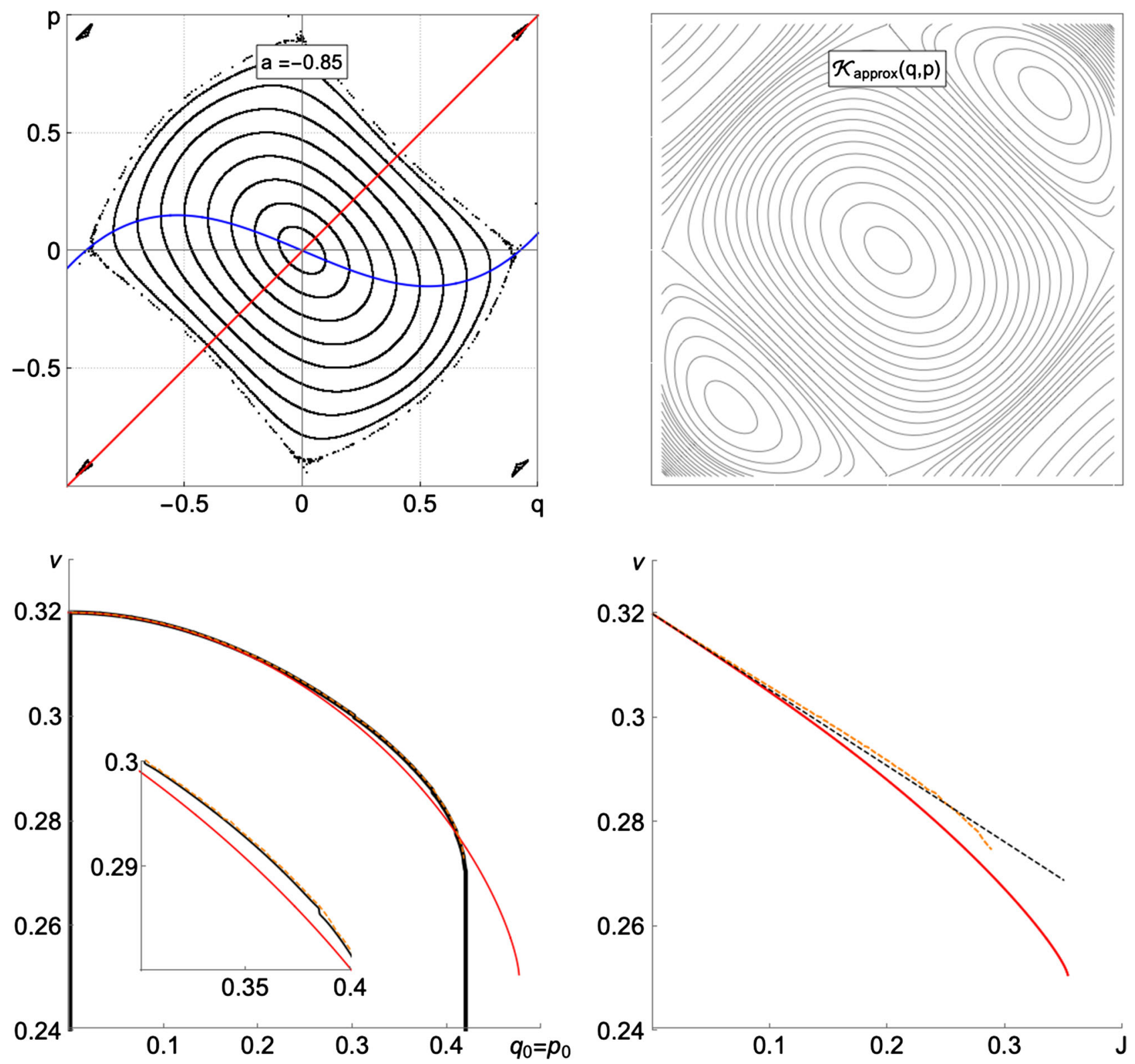

FIG. 4. Top row: Phase-space trajectories of a cubic map, obtained by tracking with $a=-0.85$ (left plot) and level sets of the approximate invariant (34) (right plot), on the same scale. The red and blue lines in the top left plot correspond to symmetry lines $p=q$ and $p=\left(a q+\epsilon q^{3}\right) / 2$ respectively. Bottom row: The left plot shows the rotation number as a function of initial conditions in the form $q_{0}=p_{0}$, by using Eq. (2) (black solid line), and by using the Danilov equation, Eq. (12) numerically (orange dashed). The red solid line corresponds to the rotation number obtained from the approximate invariant (34) by using the Danilov equation as well. The right bottom plot shows the dependence of $\nu$ as a function of action $J$, from tracking (orange dashed) and from the approximate invariant (34) (red solid).

$$
\nu(J) \approx \nu(0)-\frac{3}{2 \pi} \frac{\epsilon}{4-a^{2}} J
$$

which is the same as in [28] and similar to Eq. (31).

One can now envision using such a method sequentially, as a perturbation method, by introducing higherorder (in $\epsilon$ ) terms in the approximate integral, Eq. (34), and thus obtaining higher-order terms to the rotation number, Eq. (35), as well as to the time-independent Hamiltonian [29].

\section{4D integrable map}

In this section we will sketch out an example of how to use the Danilov equation to analyze an integrable multidimensional map. Consider the following map, which can be realized in accelerators by employing the so-called electron lens [30-32], 


$$
\left[\begin{array}{c}
x^{\prime} \\
p_{x}^{\prime} \\
y^{\prime} \\
p_{y}^{\prime}
\end{array}\right]=\left[\begin{array}{c}
\alpha_{x} x+\beta p_{x} \\
-\gamma_{x} x-\alpha_{x} p_{x}+\frac{a x^{\prime}}{b r^{\prime 2}+1} \\
\alpha_{y} y+\beta p_{y} \\
-\gamma_{y} y-\alpha_{y} p_{y}+\frac{a y^{\prime}}{b r^{\prime 2}+1}
\end{array}\right],
$$

where $r^{2}=x^{2}+y^{2}, \beta \gamma_{x}=1+\alpha_{x}^{2}, \beta \gamma_{y}=1+\alpha_{y}^{2}$, with $\alpha_{x}, \alpha_{y}, a, b$ and $\beta$ being some arbitrary parameters. This map has two integrals of motion in involution (having a vanishing Poisson bracket):

$$
L=\left(\alpha_{y}-\alpha_{x}\right) x y+\beta\left(x p_{y}-y p_{x}\right)
$$

and

$$
\mathcal{K}=\left(b+\frac{1}{r^{2}}\right) T^{2}+\beta a T+r^{2}+\frac{L^{2}}{r^{2}},
$$

where $T=\alpha_{x} x^{2}+\alpha_{y} y^{2}+\beta r p_{r}$ and $p_{r}=\left(x p_{x}+y p_{y}\right) / r$. In order to employ the Danilov equation, we must rewrite the map (36) in new variables, where this map is separated into two maps. Such variables exist by virtue of this map being integrable. We first notice that by introducing new variables,

$$
\begin{aligned}
\tilde{x} & =x / \sqrt{\beta} \\
\tilde{p}_{x} & =x \alpha_{x} / \sqrt{\beta}+p_{x} \sqrt{\beta} \\
\tilde{y} & =y / \sqrt{\beta} \\
\tilde{p}_{y} & =y \alpha_{y} / \sqrt{\beta}+p_{y} \sqrt{\beta},
\end{aligned}
$$

the map (36) becomes symmetric in $\tilde{x}$ and $\tilde{y}$ with $\tilde{a}=a \sqrt{\beta}$ and $\tilde{b}=b \beta$. The resulting map is separable in polar coordinates, $r$ and $\theta$, such that $x=r \cos (\theta)$ and $y=r \sin (\theta)$, where we omitted the tilde ( $)$ sign for clarity. The resulting map is

$$
\left[\begin{array}{c}
r^{\prime} \\
p_{r}^{\prime} \\
\theta^{\prime} \\
p_{\theta}^{\prime}
\end{array}\right]=\left[\begin{array}{c}
\sqrt{p_{r}^{2}+\frac{p_{\theta}^{2}}{r^{2}}} \\
-p_{r} \frac{r}{r^{\prime}}+\frac{a r^{\prime}}{b r^{\prime 2}+1} \\
\theta+\arctan \frac{p_{\theta}}{r p_{r}} \\
p_{\theta}
\end{array}\right],
$$

where the angular momentum $p_{\theta}=x p_{y}-y p_{x}=$ const is the integral of the motion. An additional integral is

$$
\mathcal{K}\left(r, p_{r}, p_{\theta}\right)=b r^{2} p_{r}^{2}+r^{2}+p_{r}^{2}-a r p_{r}+\frac{p_{\theta}^{2}}{r^{2}} .
$$

Now we will use the Danilov equation to obtain two unknown rotation numbers, $\nu_{\theta}$ and $\nu_{r}$. We first notice that $\mathcal{K}$ does not depend on $\theta$ and thus can be used to evaluate $\nu_{r}$ in Eq. (11) directly, by treating $p_{\theta}$ as a parameter:

$$
\begin{aligned}
\nu_{r}\left(\mathcal{K}, p_{\theta}\right) & =\frac{\tau}{T_{r}}=\frac{\int_{r}^{r^{\prime}}\left(\frac{\partial \mathcal{K}}{\partial p_{r}}\right)^{-1} \mathrm{~d} r}{\oint\left(\frac{\partial \mathcal{K}}{\partial p_{r}}\right)^{-1} \mathrm{~d} r} \\
& =\mathrm{F}\left[\arcsin \sqrt{\frac{\zeta_{3}-\zeta_{1}}{\zeta_{3}+1}}, \kappa\right] /[2 \mathrm{~K}(\kappa)],
\end{aligned}
$$

where $\mathrm{K}(\kappa)$ is the complete elliptic integral of the first kind, $\mathrm{F}(\phi, \kappa)$ is the incomplete elliptic integral of the first kind, elliptic modulus $\kappa$ is given by

$$
\kappa=\sqrt{\frac{\zeta_{3}-\zeta_{2}}{\zeta_{3}-\zeta_{1}}}
$$

and $\zeta_{1}<0<\zeta_{2}<\zeta_{3}$ are the roots of the polynomial

$$
\mathcal{P}_{3}(\zeta)=-\zeta^{3}+\left[\mathcal{K}+\left(\frac{a}{2}\right)^{2}-1\right] \zeta^{2}+\left(\mathcal{K}-p_{\theta}^{2}\right) \zeta-p_{\theta}^{2}
$$

In order to evaluate the angular rotation number, $\nu_{\theta}$, we first notice that there is some uncertainty as to which integral of the motion to employ: one can add an arbitrary function of $p_{\theta}$ to $\mathcal{K}, \mathcal{K}^{\prime}=\mathcal{K}+f\left(p_{\theta}\right)$, to obtain another integral. This new integral of motion, $\mathcal{K}^{\prime}$, gives the same $\nu_{r}$, but modifies the angular motion by some unknown linear function of time:

$$
\begin{aligned}
& \frac{d \theta}{d t}=\frac{d \mathcal{K}^{\prime}}{d p_{\theta}}=\frac{d \mathcal{K}}{d p_{\theta}}+f^{\prime}\left(p_{\theta}\right), \\
& \theta(t)=\int \frac{d \mathcal{K}}{d p_{\theta}} d t+f^{\prime}\left(p_{\theta}\right) t .
\end{aligned}
$$

Fortunately, we can resolve this uncertainty by using the angular portion of the map, Eq. (40). By its definition, the angular rotation number is

$$
\nu_{\theta}=\nu_{r} \frac{\Delta \theta\left(T_{r}\right)}{2 \pi}
$$

where

$$
\Delta \theta\left(T_{r}\right)=\oint \frac{d \mathcal{K}}{d p_{\theta}}\left(\frac{\partial \mathcal{K}}{\partial p_{r}}\right)^{-1} d r+k T_{r}
$$

$k$ is an unknown coefficient and $T_{r}$ is the period of the radial motion,

$$
T_{r}=\oint\left(\frac{\partial \mathcal{K}}{\partial p_{r}}\right)^{-1} \mathrm{~d} r
$$

To determine the coefficient $k$ we will notice from Eq. (40) that $\Delta \theta(\tau)=\arctan \left(\frac{p_{\theta}}{r p_{r}}\right)$. Thus, 

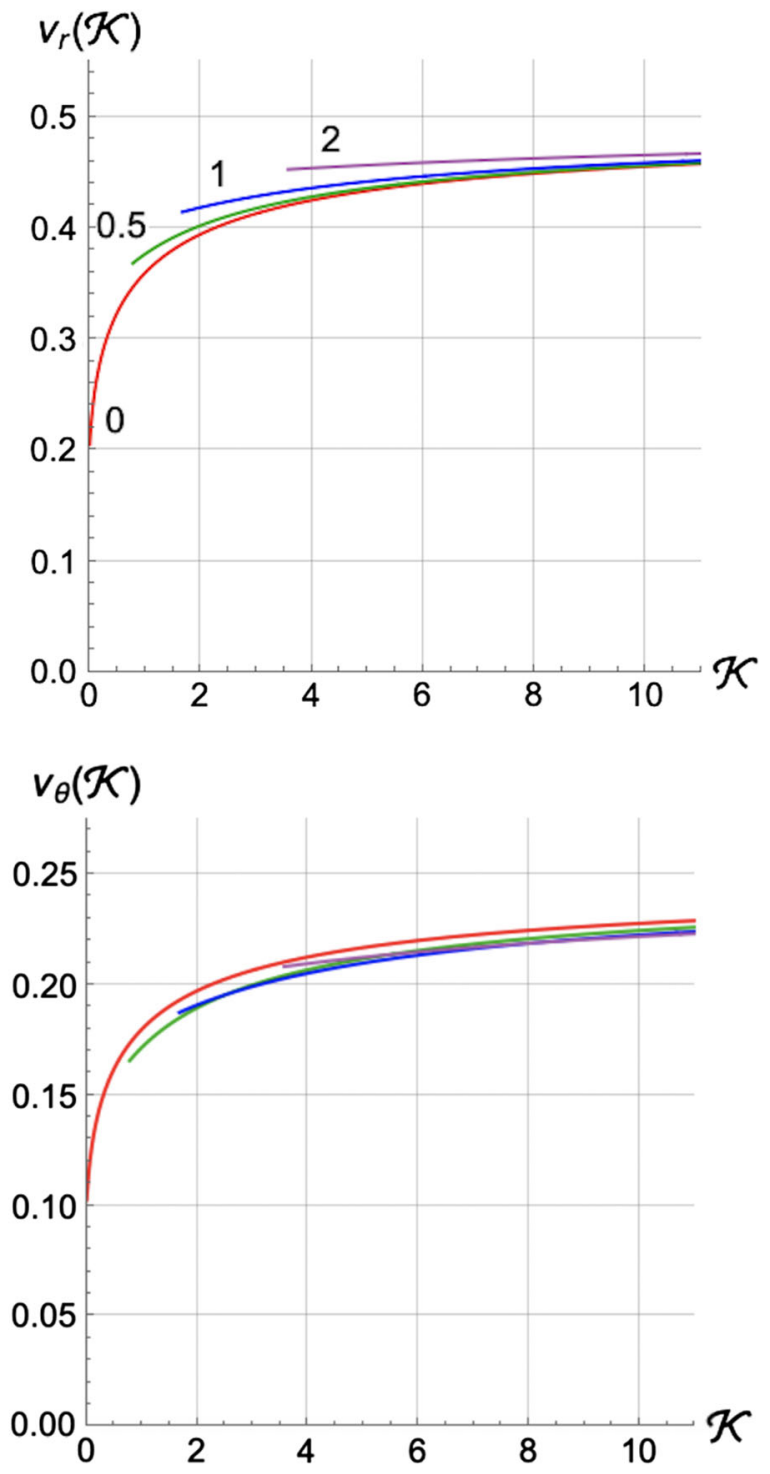

FIG. 5. Radial (top) and angular (bottom) rotation numbers as a function of the first integral of the map, $\mathcal{K}$, for different values of its second integral, $p_{\theta}$ (shown with color labels). The map parameters are $b=1$ and $a=1.6$. Note that for $p_{\theta}=0$, $\nu_{r}=2 \nu_{\theta}$, as expected, and equals the frequency $\nu$ from the one-dimensional example of Fig. 3.

$$
k=\frac{1}{\tau}\left[\arctan \left(\frac{p_{\theta}}{r p_{r}}\right)-\int_{r}^{r^{\prime}} \frac{d \mathcal{K}}{d p_{\theta}}\left(\frac{\partial \mathcal{K}}{\partial p_{r}}\right)^{-1} d r\right]
$$

with

$$
\tau=\int_{r}^{r^{\prime}}\left(\frac{\partial \mathcal{K}}{\partial p_{r}}\right)^{-1} \mathrm{~d} r
$$

Now, recalling that $\nu_{r}=\tau / T_{r}$, we finally obtain

$$
\begin{aligned}
\nu_{\theta}= & \frac{\nu_{r}}{2 \pi} \oint \frac{d \mathcal{K}}{d p_{\theta}}\left(\frac{\partial \mathcal{K}}{\partial p_{r}}\right)^{-1} d r \\
& +\frac{1}{2 \pi}\left[\arctan \left(\frac{p_{\theta}}{r p_{r}}\right)-\int_{r}^{r^{\prime}} \frac{d \mathcal{K}}{d p_{\theta}}\left(\frac{\partial \mathcal{K}}{\partial p_{r}}\right)^{-1} d r\right] .
\end{aligned}
$$

After some math, this expression can be rewritten as

$$
\nu_{\theta}\left(\mathcal{K}, p_{\theta}\right)=\frac{\Delta}{2 \pi}\left[\nu_{r}-\frac{\Delta^{\prime}}{\Delta}+\frac{\arctan \left(\frac{2 p_{\theta}}{a} \frac{\zeta_{3}+1}{\zeta_{3}}\right)}{\Delta}\right],
$$

where

$$
\begin{aligned}
\Delta & =\frac{2 p_{\theta}}{\zeta_{3} \sqrt{\zeta_{3}-\zeta_{1}}} \Pi\left[\kappa \mid \frac{\zeta_{3}-\zeta_{2}}{\zeta_{3}}\right], \\
\Delta^{\prime} & =\frac{p_{\theta}}{\zeta_{3} \sqrt{\zeta_{3}-\zeta_{1}}} \Pi\left[\arcsin \sqrt{\frac{\zeta_{3}-\zeta_{1}}{\zeta_{3}+1}}, \kappa \mid \frac{\zeta_{3}-\zeta_{2}}{\zeta_{3}}\right],
\end{aligned}
$$

and $\Pi(\kappa \mid \alpha)$ and $\Pi(\phi, \kappa \mid \alpha)$ are the complete and the incomplete elliptic integrals of the third kind, respectively. One can note that for a linear 4D map $(b=0)$, we have $\nu_{r}=2 \nu_{\theta}$ for any value of $p_{\theta}$. Figure 5 shows an example of the radial and the angular rotation numbers as a function of $\mathcal{K}$ for various values of $p_{\theta}$.

\section{SUMMARY}

In this paper we demonstrated a general and exact method of how to find a Poincaré rotation number for integrable symplectic maps of a plane and its connection to accelerator physics. It complements the discrete Arnold-Liouville theorem for maps $[21,22]$ and permits the analysis of dynamics for integrable systems. Equation (18) also permits to express the Hamilton function of a given integrable map explicitly. Several examples were presented in our paper. These examples demonstrate that the Danilov equation is a powerful tool. The McMillan integrable map is a classic example of a nonlinear integrable discrete-time system, which finds applications in many areas of physics, including accelerators $[32,33]$. It is a typical member of a wide class of area-preserving transformations called a twist map [23]. For nonintegrable maps, which are also very common in accelerator science, this new equation could allow for an approximate evaluation of rotation numbers, provided there exists an approximate integral of motion, like Eq. (34).

\section{ACKNOWLEDGMENTS}

The authors would like to thank Jeffrey Holmes and Stanislav Baturin for carefully reading this manuscript and for their helpful comments. This research is supported by Fermi Research Alliance, LLC under Contract 
No. DE-AC02-07CH11359 with the U.S. Department of Energy and by the University of Chicago.

\section{APPENDIX A: LINEAR MAPS}

In this Appendix we will consider two examples of linear maps and we will use Eq. (6) for one and Eq. (17) for the second one.

\section{Linear accelerator map}

Consider a linear symplectic map,

$$
\left[\begin{array}{l}
q^{\prime} \\
p^{\prime}
\end{array}\right]=\left[\begin{array}{ll}
a & b \\
c & d
\end{array}\right]\left[\begin{array}{l}
q \\
p
\end{array}\right]
$$

with $a d-b c=1$ and $|a+d| \leq 2$. This map is very common in accelerator physics and has been described in [2]. The rotation number (the betatron frequency) for this map is well known:

$$
\nu=\frac{1}{2 \pi} \arccos \frac{a+d}{2} .
$$

To obtain this equation using the Danilov equation, we will recall that this map has the following Courant-Snyder integral (invariant):

$$
\mathcal{K}=c q^{2}+(d-a) q p-b p^{2} .
$$

Let us assume that $c>0$, then $b \leq 0$ and $\mathcal{K}(q, p) \geq 0$ for any $q$ and $p$. From this, we obtain

$$
\left(\frac{\partial \mathcal{K}}{\partial p}\right)^{-1}=\frac{\partial p}{\partial \mathcal{K}}=\frac{ \pm 1}{\sqrt{\left[(a+d)^{2}-4\right] q^{2}-4 b \mathcal{K}}} .
$$

We will use

$$
(q, p)=(\sqrt{\mathcal{K} / c}, 0)
$$

and

$$
\left(q^{\prime}, p^{\prime}\right)=(b \sqrt{\mathcal{K} / c}, \sqrt{\mathcal{K} c}) .
$$

After a straightforward evaluation of integrals in Eq. (6), we obtain

$$
\nu=\frac{1}{2 \pi} \arccos \frac{a+d}{2},
$$

the same is in Eq. (A2).

\section{Brown map}

As a second example we will consider the Brown map [34,35], $\mathrm{M}_{\mathrm{B}}$, (a)

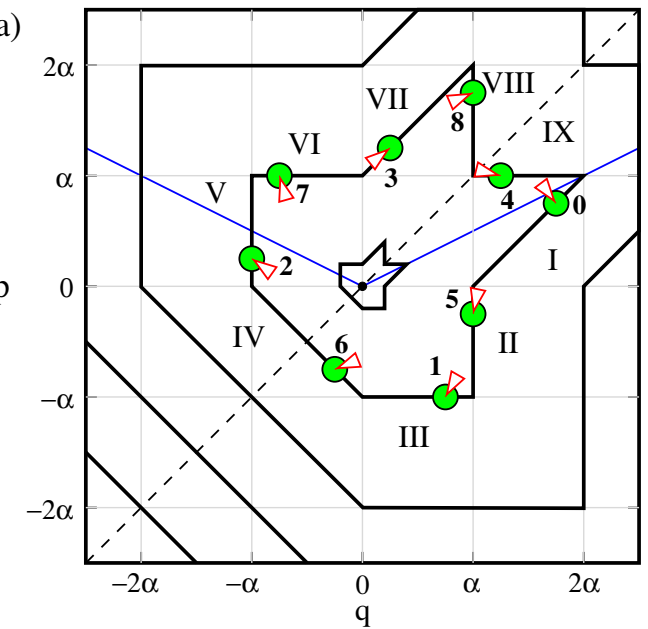

(b)
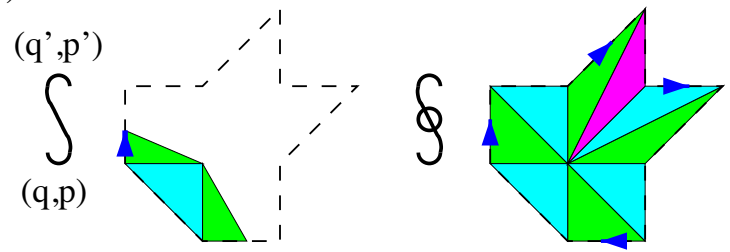

FIG. 6. Brown map. (a) Constant level sets of the invariant, $\mathcal{K}(q, p)=$ const (black solid polygons). Dashed black line $p=q$ and blue line $p=\frac{1}{2}|q|$ illustrate two reflection symmetries of the invariant polygons. Line segments are labeled with roman numerals. Green points are an example of a 9-cycle orbit, where the arabic numerals show the iteration number. (b) An example of possible contour of integration for the numerator and denominator in Danilov equation.

$$
\left[\begin{array}{c}
q^{\prime} \\
p^{\prime}
\end{array}\right]=\left[\begin{array}{c}
p \\
-q+|p|
\end{array}\right]
$$

which has the following integral:

$$
\begin{aligned}
\mathcal{K}(q, p)= & \frac{1}{8}(q+p+|q-| p||+|p-| q|| \\
& +2|q-| p-|q|||+2|p-| q-|p||| \\
& +|q-| p|+| p-|q-| p|||| \\
& +|p-| q|+| q-|p-| q||||) .
\end{aligned}
$$

The map has only one stable fixed point, located at the origin, with $\mathcal{K}=0$. Constant level sets of $\mathcal{K}>0$ are polygons, geometrically similar to each other, with nine sides, labeled by Roman numerals, see Fig. 6(a). All orbits belonging to these levels are periodic with

$$
\mathrm{M}_{\mathrm{B}}^{9}(q, p)=(q, p),
$$

and in fact, they are permutation 9-cycles such that 


$$
\begin{aligned}
\cdots \rightarrow \mathrm{I} & \rightarrow \mathrm{III} \rightarrow \mathrm{V} \rightarrow \mathrm{VII} \rightarrow \mathrm{IX} \rightarrow \\
& \rightarrow \mathrm{II} \rightarrow \mathrm{IV} \rightarrow \mathrm{VI} \rightarrow \mathrm{VIII} \rightarrow \mathrm{I} \rightarrow \cdots .
\end{aligned}
$$

Since it is a linear map ( $\nu=$ const for all orbits), we will use Eq. (17) to determine its rotation number. It is obvious from Fig. 6(b) that $J=4.5 \alpha$, while $J^{\prime}=1 \alpha$, where $\alpha$ is some arbitrary scale parameter, resulting in $\nu=\frac{2}{9}$.

\section{APPENDIX B: NUMERICAL PROCEDURE FOR DANILOV EQUATION}

In this Appendix we will consider two numerical procedures, which can be employed in order to use Eq. (12) for mappings in McMillan form when only the mapping equation is known or when we have an approximate (or an exact) invariant of the motion but we cannot compute action integrals analytically.

We will start with the case when we have only the mapping equations. As a first step we will rewrite the map in polar coordinates:

$$
q=r \cos \phi, \quad p=r \sin \phi .
$$

Then we will iterate for various initial conditions $q_{\text {ini }}^{(k)}$, let us say in a form of $q_{\text {ini }}^{(k)}=q_{0}^{(k)}=p_{0}^{(k)}$, so that we have a collection of points in a form

$$
\left(r_{0}^{(k)}, \phi_{0}^{(k)}\right),\left(r_{1}^{(k)}, \phi_{1}^{(k)}\right),\left(r_{2}^{(k)}, \phi_{2}^{(k)}\right), \ldots,\left(r_{n}^{(k)}, \phi_{n}^{(k)}\right) .
$$

We can then sort each orbit such that

$$
\tilde{\phi}_{0}^{(k)}<\tilde{\phi}_{1}^{(k)}<\tilde{\phi}_{2}^{(k)}<\cdots<\tilde{\phi}_{n}^{(k)},
$$

where $\left(\tilde{r}_{i}^{(k)}, \tilde{\phi}_{i}^{(k)}\right)$ are the points of a new sorted $k$ th orbit. Now, for each orbit we can compute the action and the partial action numerically as

$$
J^{(k)}=\frac{1}{2 \pi} \sum_{i=0}^{n} \frac{\left(\tilde{r}_{i}^{(k)}\right)^{2}}{2}\left[\tilde{\phi}_{i}^{(k)}-\tilde{\phi}_{i-1}^{(k)}\right]
$$

and

$$
J^{\prime(k)}=\frac{1}{2 \pi} \sum_{\pi / 2<\tilde{\phi}_{i}^{(k)}<\pi} \frac{\left(\tilde{r}_{i}^{(k)}\right)^{2}}{2}\left[\tilde{\phi}_{i}^{(k)}-\tilde{\phi}_{i-1}^{(k)}\right]
$$

respectively. Finally, using the Danilov equation, we can find the rotation number as a numerical derivative:

$$
\nu^{(k)}=\frac{J^{(k+1)}-J^{(k)}}{J^{(k+1)}-J^{(k)}} .
$$

If one would like to apply the Danilov equation directly to an approximate or exact invariant of motion, we can proceed in a similar manner. First, we rewrite the invariant of motion in polar coordinates, $\mathcal{K}_{\text {approx }}(r, \phi)$. Then, for different values $\mathcal{K}_{\text {approx }}^{(k)}$ we will numerically solve $n$ equations

$$
\mathcal{K}_{\text {approx }}\left(r, \phi_{i}^{(k)}\right)=\mathcal{K}_{\text {approx }}^{(k)}
$$

with $\phi_{i}^{(k)}=2 \pi i / n$ and $i=0,1, \ldots, n-1$. Denoting the smallest positive root of equation above as $r_{i}^{(k)}$, we can find action and partial actions as

$$
J^{(k)}=\frac{1}{2 \pi} \sum_{i=0}^{n-1} \frac{\left(r_{i}^{(k)}\right)^{2}}{2}\left[\phi_{i}^{(k)}-\phi_{i-1}^{(k)}\right]
$$

and

$$
J^{\prime(k)}=\frac{1}{2 \pi} \sum_{\pi / 2<\phi_{i}^{(k)}<\pi} \frac{\left(r_{i}^{(k)}\right)^{2}}{2}\left[\phi_{i}^{(k)}-\phi_{i-1}^{(k)}\right],
$$

along with the rotation number

$$
\nu^{(k)}=\frac{J^{(k+1)}-J^{(k)}}{J^{(k+1)}-J^{(k)}} .
$$

[1] D. W. Kerst and R. Serber, Electronic orbits in the induction accelerator, Phys. Rev. 60, 53 (1941).

[2] E. D. Courant and H. S. Snyder, Theory of the alternatinggradient synchrotron, Ann. Phys. 3, 1 (1958).

[3] H. Poincaré, Sur les courbes définies par les équations différentielles (III), J. Math Pures Appl. 4e Ser. 1, 167 (1885).

[4] E. Todesco, in Analysis and Modelling of Discrete Dynamical Systems, edited by D. Benest and C. Froeschle (Taylor \& Francis, London, 1998).

[5] Y. Papaphilippou, Detecting chaos in particle accelerators through the frequency map analysis method, Chaos 24, 024412 (2014).

[6] H. S. Dumas and J. Laskar, Global Dynamics and Longtime Stability in Hamiltonian Systems via Numerical Frequency Analysis, Phys. Rev. Lett. 70, 2975 (1993).

[7] A. Bazzani, E. Todesco, G. Turchetti, and G. Servizi, A normal form approach to the theory of nonlinear betatronic motion, CERN Report No. CERN-94-02, 1994.

[8] G. Turchetti and F. Panichi, Birkhoff normal forms and stability indicators for betatronic motion, in Nonlinear Dynamics and Collective Effects in Particle Beam Physics (World Scientific, Singapore, 2019), pp. 47-69.

[9] E. M. McMillan, in Topics in Modern Physics, a Tribute to E.V. Condon, edited by E. Brittin and H. Odabasi (Colorado Associated University Press, Boulder, CO, 1971), pp. 219-244.

[10] C. C. Chow and J. R. Cary, Integrable Nonlinear Accelerator Lattices, Phys. Rev. Lett. 72, 1196 (1994). 
[11] V. Danilov, Practical solutions for nonlinear accelerator lattice with stable nearly regular motion, Phys. Rev. ST Accel. Beams 11, 114001 (2008).

[12] V. Danilov and S. Nagaitsev, Nonlinear accelerator lattices with one and two analytic invariants, Phys. Rev. ST Accel. Beams 13, 084002 (2010).

[13] V. Danilov and S. Nagaitsev, Accelerator-feasible $N$-body nonlinear integrable system, Phys. Rev. ST Accel. Beams 17, 124402 (2014).

[14] A. Lichtenberg and M. Lieberman, Regular and Chaotic Dynamics (Springer-Verlag, New York, 1992).

[15] S. S. Abdullaev, in Construction of Mappings for Hamiltonian Systems and Their Applications (Springer, Berlin, 2006).

[16] R. D. Ruth, in Proceedings, 1985 SLAC Summer School on the Physics of High-energy Particle Accelerators and 1984 U.S. Summer School on High-Energy Particle Accelerators, Stanford, California, 1985 [AIP Conf. Proc. 153, 150 (1987)].

[17] J. S. Berg, R. L. Warnock, R. D. Ruth, and E. Forest, Construction of symplectic maps for nonlinear motion of particles in accelerators, Phys. Rev. E 49, 722 (1994).

[18] A. Dragt, in Handbook of Accelerator Physics and Engineering, edited by A. Chao, K. Mess, M. Tigner, and F. Zimmermann (World Scientific, Singapore, 2013), pp. 99-105.

[19] R. Dilão and R. Alves-Pires, Nonlinear Dynamics in Particle Accelerators (World Scientific Publishing, Singapore, 1996).

[20] R. S. MacKay, Renormalisation in Area-Preserving Maps (World Scientific, Singapore, 1993), Vol. 6.

[21] V. Arnold and A. Avez, Ergodic Problems of Classical Mechanics. (The Mathematical Physics Monograph Series) (W.A. Benjamin, Inc., New York, 1968).

[22] A. P. Veselov, Integrable maps, Russ. Math. Surv. 46, 1 (1991).

[23] J. D. Meiss, Symplectic maps, variational principles, and transport, Rev. Mod. Phys. 64, 795 (1992).

[24] V. Danilov (private communication).

[25] M. Glasser, V. Papageorgiou, and T. Bountis, Mel'nikov's function for two-dimensional mappings, SIAM J. Appl. Math. 49, 692 (1989).
[26] A. Iatrou and J. A. G. Roberts, Integrable mappings of the plane preserving biquadratic invariant curves II, Nonlinearity 15, 459 (2002).

[27] M. Hénon, Numerical study of quadratic area-preserving mappings, Quart. Appl. Math. 27, 291 (1969).

[28] H. Dullin and J. Meiss, Generalized Hénon maps: The cubic diffeomorphisms of the plane, Physica (Amsterdam) 143D, 262 (2000).

[29] T. Zolkin and S. Nagaitsev, Perturbation theory for symplectic mappings of the plane (to be published).

[30] V. Shiltsev, V. Danilov, D. Finley, and A. Sery, Considerations on compensation of beam-beam effects in the Tevatron with electron beams, Phys. Rev. ST Accel. Beams 2, 071001 (1999).

[31] V. Shiltsev, K. Bishofberger, V. Kamerdzhiev, S. Kozub, M. Kufer, G. Kuznetsov, A. Martinez, M. Olson, H. Pfeffer, G. Saewert, V. Scarpine, A. Seryi, N. Solyak, V. Sytnik, M. Tiunov, L. Tkachenko, D. Wildman, D. Wolff, and X.-L. Zhang, Tevatron electron lenses: Design and operation, Phys. Rev. ST Accel. Beams 11, 103501 (2008).

[32] I. Lobach, S. Nagaitsev, E. Stern, and T. Zolkin, in Proceedings of the 9th International Particle Accelerator Conference (IPAC'18), Vancouver, BC, Canada, 2018, International Particle Accelerator Conference No. 9 (JACoW Publishing, Geneva, Switzerland, 2018), pp. 3143-3145.

[33] S. Antipov, D. Broemmelsiek, D. Bruhwiler, D. Edstrom, E. Harms, V. Lebedev, J. Leibfritz, S. Nagaitsev, C. Park, H. Piekarz, P. Piot, E. Prebys, A. Romanov, J. Ruan, T. Sen, G. Stancari, C. Thangaraj, R. Thurman-Keup, A. Valishev, and V. Shiltsev, IOTA (Integrable Optics Test Accelerator): Facility and experimental beam physics program, J. Instrum. 12, T03002 (2017).

[34] M. Brown, The American mathematical monthly, Am. Math. Mon. 90, 569 (1983).

[35] M. Brown, A periodic homeomorphism of the plane, in Continuum Theory and Dynamical Systems (Lecture Notes in Pure and Applied Mathematics), edited by T. West (Marcel Dekker, Inc, New York, 1993), Vol. 149, pp. 83-87. 